\title{
Effect of Particle Size Distribution on SiC Ceramic Sinterability
}

\author{
Nur Zalikha Khalil ${ }^{1, *}$, Sanjay K. Vajpai ${ }^{2}$, Mie Ota $^{3}$ and Kei Ameyama ${ }^{3}$ \\ ${ }^{1}$ Department of Mechanical Engineering, Ritsumeikan University, Kusatsu 525-8577, Japan \\ ${ }^{2}$ Research Organization of Science and Technology, Ritsumeikan University, Kusatsu 525-8577, Japan \\ ${ }^{3}$ Faculty of Science and Engineering, Department of Mechanical Engineering, Ritsumeikan University, \\ Kusatsu 525-8577, Japan
}

Silicon Carbide has drawn a significant attention in recent decades for its excellent properties such as high oxidation resistance, high hardness and low density. However, an important drawback for $\mathrm{SiC}$ manufacturing is its poor sinter ability due to its highly covalent nature of bonding. This work investigates the effect of dispersion in Particle Size Distribution on the sinter ability of SiC ceramics. In the present work, two types of SiC powders, consisting powders of sizes $0.3 \mu \mathrm{m}$ and $2.5 \mu \mathrm{m}$, were subjected to mechanical milling in order to produce various patterns of particle size distributions. Subsequently, milled powders were sintered by spark plasma sintering. This sintering technology is used in account of its rapid heating and short time sintering. This work also suggests the usage of coefficient of variation, $C_{\mathrm{v}}$ as an appropriate parameter to indicate the dispersion level in PSD range investigated. [doi:10.2320/matertrans.Y-M2015823]

(Received July 3, 2015; Accepted August 7, 2015; Published October 9, 2015)

Keywords: silicon carbide, particle size distribution, mechanical milling, spark plasma sintering, sinterability

\section{Introduction}

Silicon Carbide $(\mathrm{SiC})$ is a typical candidate for high temperature applications owing to its excellent thermal and mechanical properties. ${ }^{1-5)}$ However, due to its highly covalent bonded characteristic and the resultant low self-diffusion coefficient, ${ }^{6,7)}$ sintering of $\mathrm{SiC}$ has been a challenging topic for materials' researcher. Generally, the conventional solid state sintering requires extremely high temperatures up to $2200^{\circ} \mathrm{C}$ and long sintering time to achieve full densification. ${ }^{7,8)}$ It is well known that high temperature and longtime sintering may cause the grain growth phenomenon that will eventually detract the mechanical properties of the final sintered product. With regard to particle size distributions (PSD), earlier researchers have suggested using mono-sized powders of narrow particle size distribution in producing uniform, dense and fine-grained microstructure. $^{9-12)}$ Later, Patterson and Griffin ${ }^{13)}$ has reported that for coarse spherical copper powder, the increasing of PSD led to an increase in densification while in fine tungsten powders, the narrowest and widest distribution made the most rapid densification compared to the intermediate one. Working on commercial alumina powder, Yeah and Sacks ${ }^{14)}$ have reported that broad distribution is preferable due to a higher starting green density. In recent work on submicron alumina powder, Ma and $\mathrm{Lim}^{15)}$ have demonstrated that for practical use, narrow PSD is preferred to monosized or broad size distribution for better sinterability due to the microstructural homogeneity of the green compact, provided that the powder is agglomerate free to start with. They also added that the optimum PSD for a given powder system would vary depending on the consolidation technique and condition used. Recently, Andreas et al. ${ }^{16)}$ have simulated the effect of PSD on solid state sintering for several patterns of PSD (normal, log-normal and bimodal). They have reported that

*Graduate Student, Ritsumeikan University. Corresponding author, E-mail: daisyrose87@yahoo.com; gr0167hv@ed.ritsumei.ac.jp polydispersity in PSD resulted in the declination of densification rate with increasing distribution width. This is due to the lower coordination number and higher average contacts area in the initial packing structure. Apparently, the effects of particle size distributions are inconsistent and rather complicated since it depends to a great extent on the sintering mechanism and material used. Thus, present work attempts to establish the effect of dispersion in particle size distribution on sinterability of $\mathrm{SiC}$ ceramic. This work also discusses the appropriate dispersion measure for distributions with different variables (e.g.: mean, standard deviation). In this work, $\mathrm{SiC}$ powders were subjected to mechanical milling in order to produce various kinds of particle size distributions. Wet milling is adopted due to its effectiveness to increase homogeneous dispersion in powder while milling is conducted. ${ }^{17)}$ Subsequently milled powders were subjected to spark plasma sintering and the sinterability of powder compacts were evaluated by measuring the relative density and flexural strength. The evolution of powders particle morphology during mechanical milling is also discussed.

\section{Experimental Procedures}

In present work, coarse $\alpha$-SiC powder of $2.5 \mu \mathrm{m}$ and a relatively fine $\mathrm{SiC}$ powder of $0.3 \mu \mathrm{m}$ commercialized by Kojundo Chemical Labroratory, Co., Ltd. were used. Each powder was subjected to vibratory ball mill (Super Misuni NEV-MA-8 Nishin Gikken, Japan). Dense tungsten carbidecobalt (WC-Co) of $11 \mathrm{~mm}$ in diameter was used as a grinding media. Wet milling was conducted in the presence of ethanol to avoid agglomeration while milling and the ratio of ethanol to powders is $1: 1$ by mass. A constant vibration frequency of $12.5 \mathrm{~Hz}$ and a total time of $0 \mathrm{ks} \sim 360 \mathrm{ks}$ were used to mill the powders and the ratio of the grinding ball to milled powder was $5: 1$ by mass. After milling, the powders were dried and sieved to eliminate hard agglomerate. Subsequently, particle size was measured using Laser Diffraction 

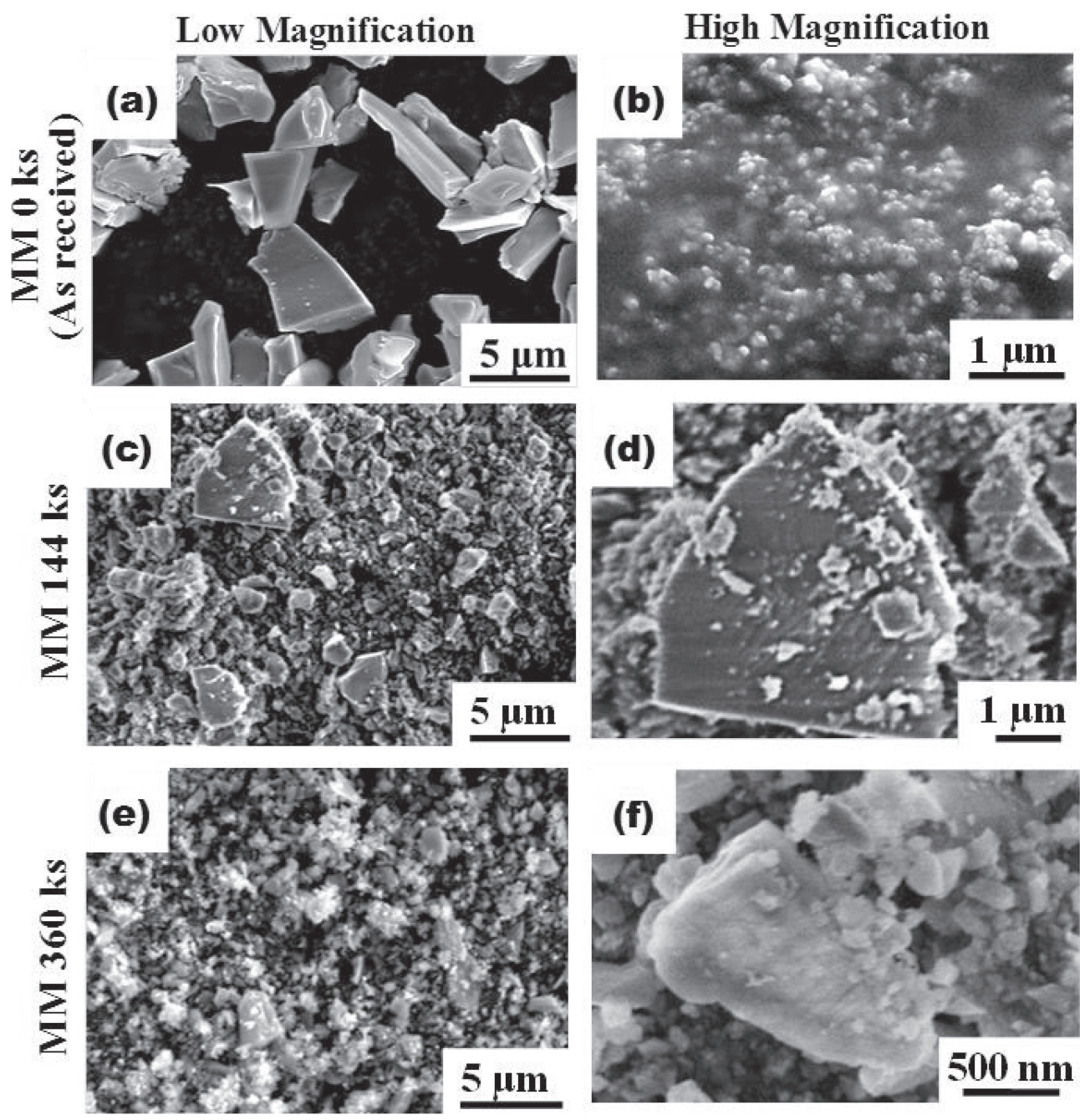

Fig. 1 SEM micrographs of coarse SiC powders in low and high magnifications (a) and (b) are as received powders, (c) and (d) are MM $144 \mathrm{ks}$ powders, (e) and (f) are MM $360 \mathrm{ks}$ powders.

Particle Size Analyser (Shidmadzu SALD-2300, Japan) using 10 different powder samples to get the average distribution pattern for each powder. The morphology of milled powders were observed using Field Emission Scanning Electron Microscope (FE-SEM S-4800, Hitachi Japan). The milled powders were inserted in a graphite die with external and internal diameter of $30 \mathrm{~mm}$ and $15.5 \mathrm{~mm}$, respectively, with graphite punch having a diameter of $15 \mathrm{~mm}$ and $20 \mathrm{~mm}$ height. Subsequently, the powders were sintered by Spark Plasma Sintering (SPS) using DR.SINTER 1020 apparatus SPS Syntex Inc., Japan at $1873 \mathrm{~K}$ for $0.6 \mathrm{ks}$ under an applied pressure of $50 \mathrm{MPa}$ and carried out in a vacuum. The temperature was measured using an infrared active homing (IR-AH) thermometer. After the powders were soaked in the desired temperature, the pressure was released and the specimen is cooled down to room temperature. Density for the sintered compacts was measured by dividing the weight of the compacts by its dimension. The sintered samples were carefully polished and microstructure characterization was conducted by means of SEM. The mechanical properties of the sintered $\mathrm{SiC}$ compacts were evaluated by 4-point bend test. The dimension of the bend test specimen was $13 \mathrm{~mm} \times 4 \mathrm{~mm} \times 2 \mathrm{~mm}$ specimens, with crosshead of $0.1 \mathrm{~mm} / \mathrm{min}$.

\section{Results}

\subsection{Effect of mechanical milling to particle morphology and PSD}

Figure 1 shows the appearance of both as received and milled coarse $\mathrm{SiC}$ powders at low and high magnifications. As received coarse powder used in this study has angular morphology (Fig. 1(a)). However, it is also interesting to note that the as received coarse $\mathrm{SiC}$ powder consisted of a significant amount of nano-sized particles also, as shown by a high magnification SEM micrograph in Fig. 1(b). Therefore, it appears that the as-received coarse $\mathrm{SiC}$ powder had particles of two different size regimes, i.e. coarse micronsized and fine nano-sized particles. After milling for $144 \mathrm{ks}$, it can be observed that coarse particles have fragmented to smaller particles as shown in Fig. 1(c). This can be attributed to the intense impact of ball while milling was conducted. It can also be observed that a wide range of particles exist after milling and the shape of the particle is somewhat a mixture of angular and partially rounded. Figure 1(d) shows the remained coarse particle after milling. It is partially rounded in morphology and the existence of many nano-sized particles after milling is also observed. Further milling for $360 \mathrm{ks}$ continues crushing $\mathrm{SiC}$ particles as shown in Fig. 1(e). 

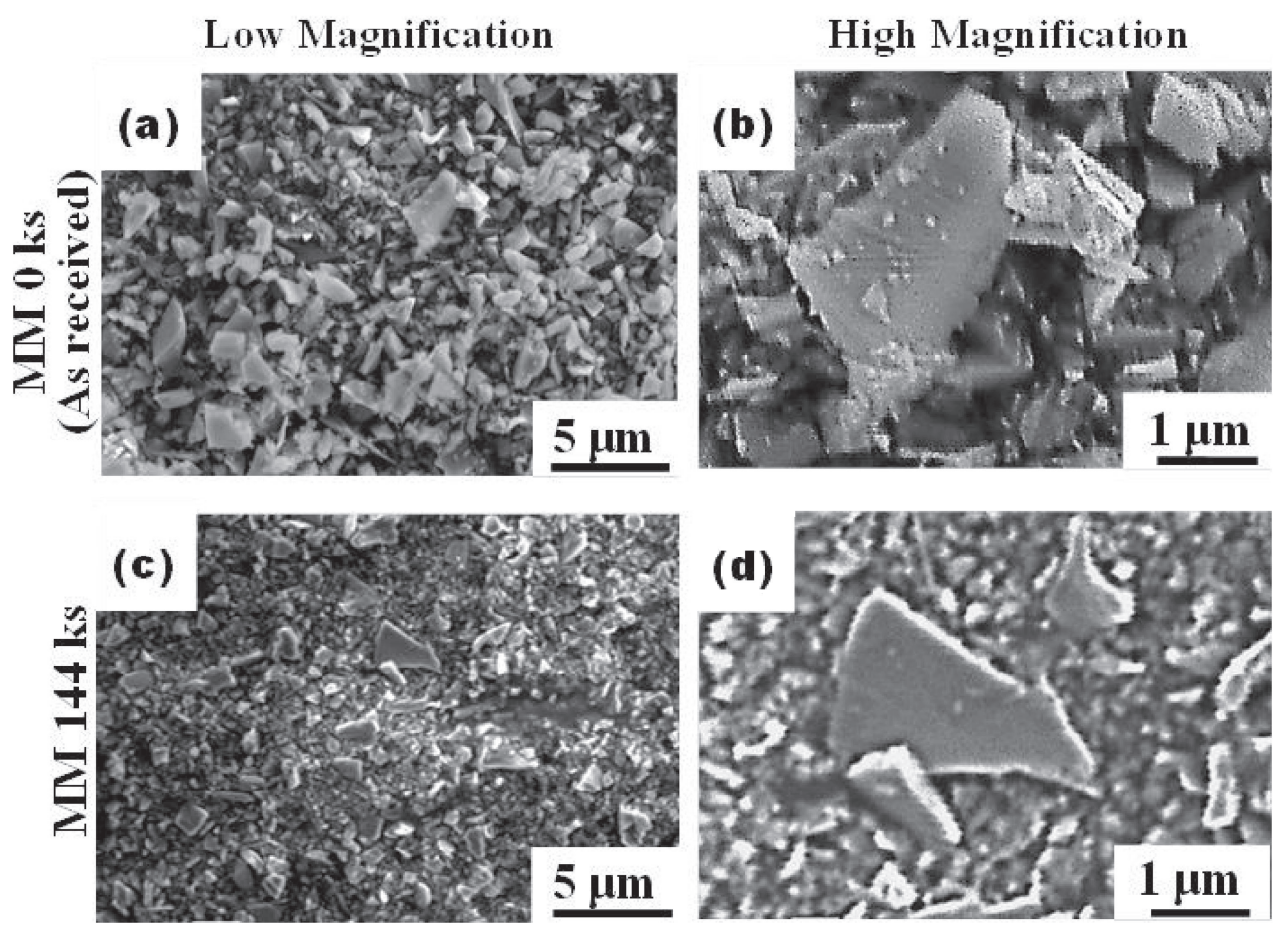

Fig. 2 SEM micrographs of fine SiC powders in low and high magnifications (a) and (b) are as received powders, (c) and (d) are MM $144 \mathrm{ks}$ powders.

As a result, most of the $\mathrm{SiC}$ particles have fragmented to nanosized particles. The final shape of the particles is somewhat rounded, as shown in Fig. 1(f). Similarly, Fig. 2 shows the appearance of both as received and milled fine $\mathrm{SiC}$ powders at low and high magnifications. As received fine powders has an angular morphology, as shown in Fig. 2(a). It would be worth mention that this powder initially consists of many nano-size particles as shown in Fig. 2(b). After milling for $144 \mathrm{ks}$, it can be observed that the fragmentation occurs to the most of the particles, as shown in Fig. 2(c). However, there is no significant morphological change as the particle remained angular, as shown in Fig. 2(d). It is worth mentioning that from these morphological observations, milled powders do not show any obvious agglomeration. This might be attributed to the adoption of wet milling using ethanol while preparing these powders. Generally, very fine-sized particles, especially nano-sized particles, tend to agglomerate due to higher interparticle attractive forces, which resist dispersion. This is undesirable because it leads to poor flow of powder during compaction, resulting in decreased packing density. However, the usage of ethanol as process control agent (PCA) acts as a dispersant to induce particle-particle repulsion, which in turn hinders the agglomeration amongst the particles. As a result, no significant agglomeration was observed in the present case.

Table 1 shows the mean particle size for both coarse and fine powder. It is observed that, for coarse powders, mechanical milling leads to a significant reduction in mean particle size with increasing milling time, and a stable mean particle size is attained after approximately $144 \mathrm{ks}$ of milling time, as no significant reduction in the mean particle size was observed after subsequent milling up to $360 \mathrm{ks}$. However, it is interesting to note that the mean particle size remains
Table 1 Mean particle size.

\begin{tabular}{ccc}
\hline \multirow{2}{*}{ Milling time $(\mathrm{ks})$} & \multicolumn{2}{c}{ Mean particle Size $(\mu \mathrm{m})$} \\
\cline { 2 - 3 } & Coarse & Fine \\
\hline 0 (As received) & 2.5 & 0.3 \\
144 & 0.6 & 0.4 \\
360 & 0.6 & \\
\hline
\end{tabular}

unchanged despite of a significant particle fragmentation observed in SEM micrographs corresponding to the powders milled for $144 \mathrm{ks}$ and $360 \mathrm{ks}$ (Figs. 1(c)-1(f)). This observation could be attributed to the presence of a significantly larger number fraction of finer particles than the coarser particles in MM $144 \mathrm{ks}$ powder, leading to no significant effect of coarser particle size on the mean particle of the aggregate powder mass. It must be emphasized at this juncture that the variation in overall size distribution is expected to have more pronounced effect on the densification rather than a slight variation in mean particle size of the powder mass aggregate. Similarly, mechanical milling resulted in no significant change in the mean particle size in case of fine-sized powders also. This might be due to the limit of particle fragmentation under present milling conditions.

Figure 3 shows the PSD for both as received and milled $\mathrm{SiC}$ coarse powders. For as received coarse powder, it apparently demonstrates a bimodal distribution with two distinct peaks, namely coarse and fine peaks, as shown in Fig. 3(a). It can be observed that, as the milling time increased to $144 \mathrm{ks}$, the frequency of the coarse particles decreased significantly and the peak corresponding to coarser 

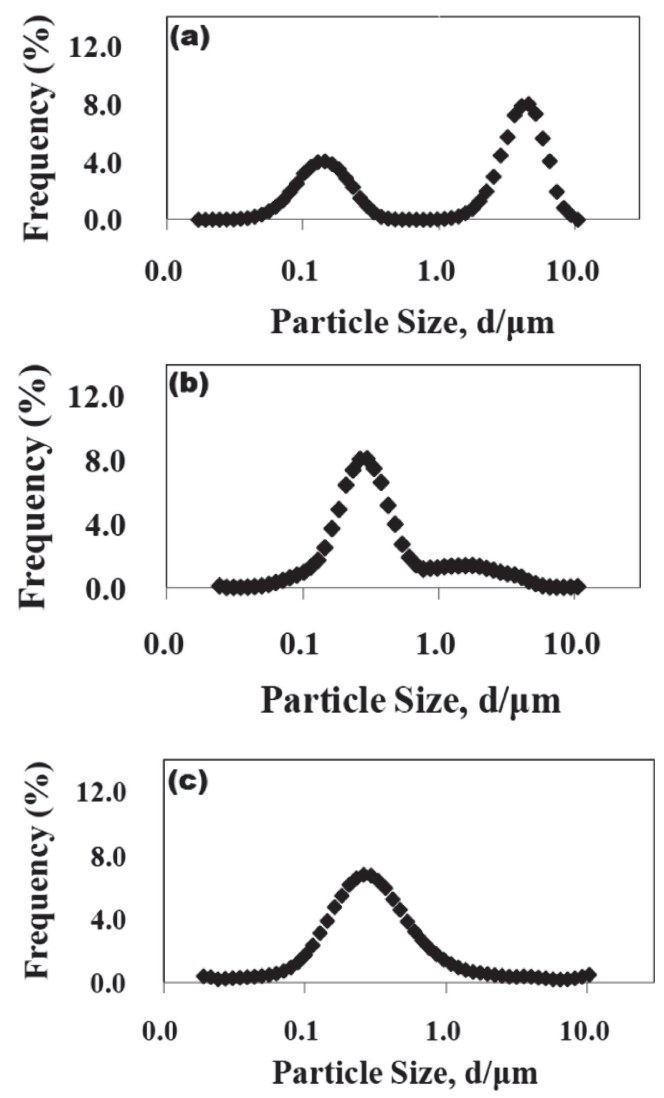

Fig. 3 Particle size distributions of coarse SiC powders; (a) As received, (b) MM $144 \mathrm{ks}$, (c) MM $360 \mathrm{ks}$.

particles shifted toward the relatively smaller particle size, as shown in Fig. 3(b). These results suggest that the milling led to the fragmentation of only the particle with size larger than $1 \mu \mathrm{m}$. It is also noted that the PSD is broadened while retaining a somewhat bimodal distribution. Subsequent milling up to $360 \mathrm{ks}$ demonstrated a complete disappearance of peak corresponding to coarser particles, as shown in Fig. 3(c), indicating that the particles with size greater than $1.0 \mu \mathrm{m}$ are further fragmented at this stage. Moreover, the PSD demonstrates a broadened unimodal distribution. It is also worth mention that the final PSD demonstrates a skewed right distribution. In general, skewed distribution is a statistical term to describe a distribution in which one of its tails is stretched out longer than the other, and the skewness direction will take the side of the longer tail. ${ }^{18)}$

Figure 4 shows the PSD for fine SiC powders. Initial fine powder had a narrow bimodal distribution, as shown in Fig. 4(a). After milling was carried out for $144 \mathrm{ks}$, the distribution became a complete unimodal distribution, as shown in Fig. 4(b). It is also observed that the PSD is somewhat broadened. These observations suggest that the fragmentation under present milling conditions only occur to the particle with size larger than $1 \mu \mathrm{m}$. The final PSD also demonstrate a skewed right distribution. Therefore, the effect of mechanical milling to particle morphology and PSD can be concluded as follows; (1) for coarse powders, milling leads to significant reduction of particle mean size and the reduction of particle aspect ratio, (2) for fine powders, milling does not reduce the mean particle size and particle aspect
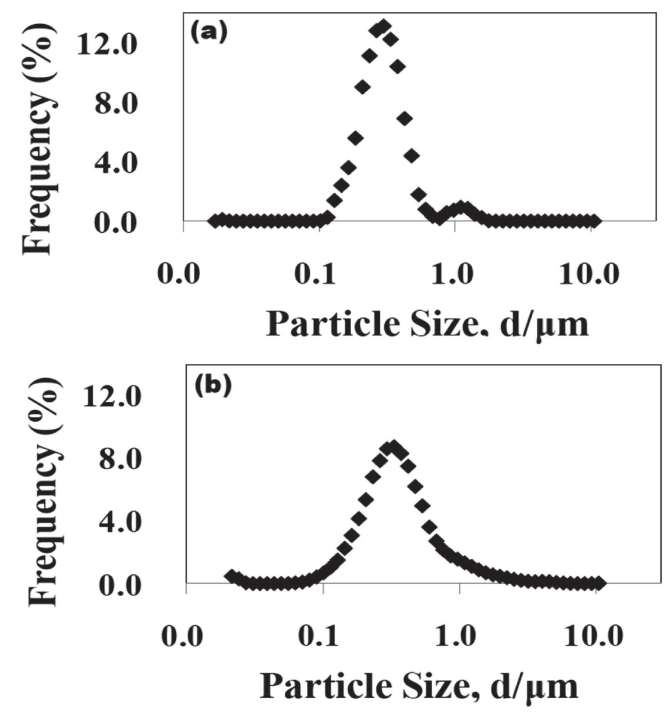

Fig. 4 Particle size distributions of fine SiC powders; (a) As received, (b) MM $144 \mathrm{ks}$.

Table 2 Relative density and mechanical properties of SiC compact.

\begin{tabular}{cccc}
\hline & $\begin{array}{c}\text { Milling } \\
\text { Time }(\mathrm{ks})\end{array}$ & $\begin{array}{c}\text { Relative } \\
\text { Density }(\%)\end{array}$ & $\begin{array}{c}\text { Flexural } \\
\text { Strength }(\mathrm{MPa})\end{array}$ \\
\hline \multirow{3}{*}{ Coarse } & 0 (As received) & $58.1 \pm 5.2$ & $105 \pm 6.3$ \\
& 144 & $75.3 \pm 4.3$ & $187 \pm 26.9$ \\
& 360 & $81.8 \pm 6.5$ & $219 \pm 43.1$ \\
\multirow{2}{*}{ Fine } & 0 (As received) & $68.6 \pm 3.3$ & $171 \pm 28.3$ \\
& 144 & $67.6 \pm 2.5$ & $163 \pm 21.1$ \\
\hline
\end{tabular}

ratio significantly, (3) mechanical milling leads to the fragmentation of particles with size greater than $1 \mu \mathrm{m}$, resulting in a broadened unimodal size distribution for both coarse and fine powders.

\subsection{Relative density and mechanical properties}

Table 2 shows the relative density and flexural strength of the powder compacts with its standard deviation. The average density of sintered compacts was evaluated by the quotient of the weight of compact and its dimensions. The flexural strength was obtained by 4-point bend test using three bars for each powder condition. It can be observed that, for coarse powder compacts, the relative density and flexural strength is increasing as milling time increases. This may be attributed to the increase in specific surface area in mechanically milled powders which stores more surface energy and sinters quickly. Moreover, powders obtained from high-energy ball-milling consist of various particle sizes, leading to easier filling of pores and providing shorter diffusion distances for atoms, which is necessary for achieving higher densification; ${ }^{19,20)}$ On the other hand, for fine powder compacts, it is observed that the relative density and flexural strength has not significantly changed as milling time increases. This might be due to the limit of fragmentation for $\mathrm{SiC}$ particle under present milling condition, which results in no significant change in surface energy and densification rate as explained beforehand. 


\section{Discussions}

\subsection{Dispersion measures in PSD}

\subsubsection{Variance and standard deviation}

In order to define dispersion level in PSD, typical dispersion measures for frequency distribution which are sample standard deviation, $s$ and sample variance, $s^{2}$ are calculated using eqs. (1) and (2), respectively; ${ }^{18)}$

Sample standard deviation,

$$
s=\sqrt{\Sigma x^{2} f-\left((\Sigma x f)^{2} / \Sigma f\right) / \Sigma f-1}
$$

Sample variance,

$$
s^{2}=\Sigma x^{2} f-\left((\Sigma x f)^{2} / \Sigma f\right) / \Sigma f-1
$$

In these equations, $x$ refers to the particle size and $f$ indicates the frequency of particle on the specific $x$ value.

\subsubsection{Coefficient of variation, $\boldsymbol{C}_{\mathrm{v}}$}

As mentioned at 4.1.1, variance and standard deviation are the most commonly used dispersion measures in statistic. When compared to the variance, the standard deviation has an important advantage: it is expressed in the same units as the variable under study, while the variance is measured in the square units of the respective variable making the standard deviation is easier to interpret. However, when the main purpose is to compare the dispersion of distributions of several variables, standard deviation is not the most appropriate indicator unless all the variables are expressed in the same measurement units and have identical mean values. When these two requirements do not hold, the coefficient of variation, $C_{\mathrm{v}}$ which measure relative dispersion expressed as the standard deviations a proportion of the arithmetic mean is frequently used. The equation is shown in eq. (3);

$$
\text { Coefficient of variation, } C_{\mathrm{v}}=s / \bar{X}
$$

where $s$ and $\bar{X}$ represent sample standard deviation and sample mean respectively. $\bar{X}$ value is calculated using the following equation;

$$
\text { Sample mean, } \bar{X}=\Sigma x f / n
$$

where $n=\Sigma f$.

The variable with largest value for the $C_{\mathrm{V}}$ is the one with the highest relative dispersion around mean. It is important to note that the ratio only applies when the expected values is not zero or negative. Moreover, it is worth mentioning that $C_{\mathrm{v}}$ is a normalized statistical measure which make it dimensionless and useful for comparing relative variability of strictly positive random variable distributions. ${ }^{21,22)}$ Therefore, in this work, $C_{\mathrm{v}}$ is introduced as an alternative dispersion measure for PSD, considering the mean size of PSD range investigated is not identical.

\subsection{Correlation between PSD parameters and sinter- ability}

Figure 5 shows the relation of relative density when plotted using three main dispersion parameters which are standard deviation $s$, variance $s^{2}$ and coefficient of variation $C_{\mathrm{v}}$. Similarly, Fig. 6 shows the relation of flexural strength when plotted with those dispersion parameters. It is worth to mention that relative density and flexural strength are used as an indicator to determine the accuracy of the dispersion
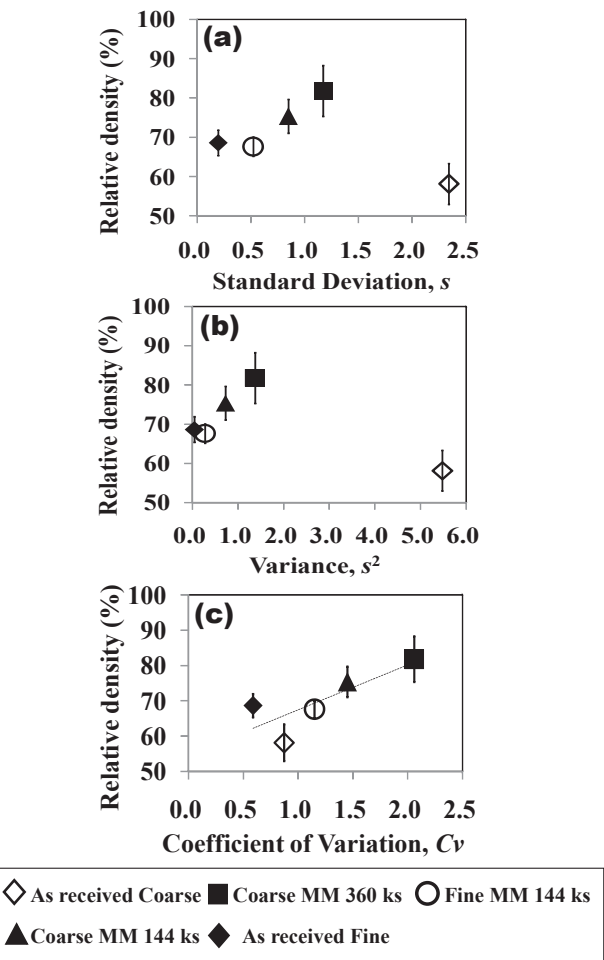

Fig. 5 Relative density plotted with dispersion measures; (a) standard deviation, (b) variance, (c) coefficient of variation.
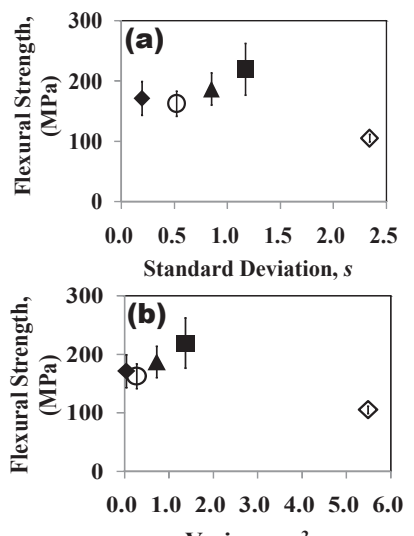

Variance, $s^{2}$

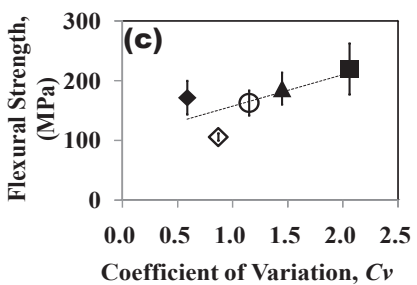

$\diamond$ As received Coarse Coarse MM $360 \mathrm{ks}$ O Fine MM $144 \mathrm{ks}$
$\Delta$ Coarse MM $144 \mathrm{ks}$ As received Fine

Fig. 6 Flexural Strength plotted with dispersion measures; (a) standard deviation, (b) variance, (c) coefficient of variation.

parameters. It is well known that the flexural strength is dependent on the relative density. Higher relative density provides stronger interparticle bonding, which hinders crack propagation and leads to higher flexural strength. From these result, it is observed that when the experimental results are plotted using powder standard deviation, $s$, and variance, $s^{2}$, no clear and reliable correlation between these parameters 
and experimental results is observed. As already suggested in 4.1.2, standard deviation and variance are not the appropriate parameter for indicating dispersion when comparing distribution with different mean size. It is a normal practice to set the mean particle size constant when comparing dispersion level in several powders so that the dispersion measure value will be reliable. On the other hand, the experimental results show a positive linear relation when plotted using coefficient of variation, $C_{\mathrm{v}}$. As proposed in 4.1.2, at this point, higher $C_{\mathrm{v}}$ value (i.e.: higher relative dispersion) is expected to lead to a higher relative density and flexural strength, attributed to the improvement in packing density due to the gap filling ability of finer particles. This leads to improvement in particle rearrangement and enhance particle average coordination number, which favors subsequent sintering. As mentioned in the introduction of the present paper, several significant efforts have been made by various researchers to determine the effect of dispersion in PSD to the sinterability. ${ }^{9-15)}$ However, in these research works, the mean particle size had to be made constant before comparing the dispersion of powders. Obviously, it is not always practically feasible, especially for commercial powders. In this respect, $C_{\mathrm{v}}$, as normalized dispersion measure has demonstrated a convenient solution to this problem by providing a more reliable measure when comparing the dispersion level in powders having different mean particle sizes. Therefore, these results justify the usage of $C_{\mathrm{V}}$ as the appropriate dispersion measure, in the PSD range investigated, as it is comparatively accurate in describing the relation between dispersion in initial powder and sintering behavior.

\subsection{Relationship between dispersion in PSD with sinter- ability}

Figure 7 shows the microstructure of as received fine and milled $360 \mathrm{ks}$ coarse powder compact. The $C_{\mathrm{V}}$ value for those initial powders are 0.59 and 2.06 , respectively. It is observed that for as received fine powder compact, the sintered region is very minimum, as shown in Fig. 7(a). Moreover, the existence of many pores can also be noted, which suggest the poor sinterability in this compact. On the other hand, for milled $360 \mathrm{ks}$ coarse compact, an improvement in sinterability is observed when the sintered region is significantly increased, as shown in Fig. 7(b). These results indicate that under a constant sintering temperature, higher $C_{\mathrm{v}}$ value of initial powder results in improved sinterability. In general, sinterability of a powder compact is determined by several factors, namely mean particle size, size distribution, and packing structure. Considering the mean particle size, particles with smaller mean size sinter quickly as compared to the relatively coarser particles. The better sintering characteristic of the relatively smaller particles can be attributed to the larger specific surface area of the smaller particles which leads to increased surface energy and favors subsequent sintering. However, it is inadequate to consider this factor individually because the dispersion of the PSD also plays important role in determining the initial packing structures as explained beforehand. It is often difficult to predict the sinterability of powders accurately when considering several powders having different mean particle sizes and dispersion. Thus, by applying the $C_{\mathrm{v}}$ value, which takes
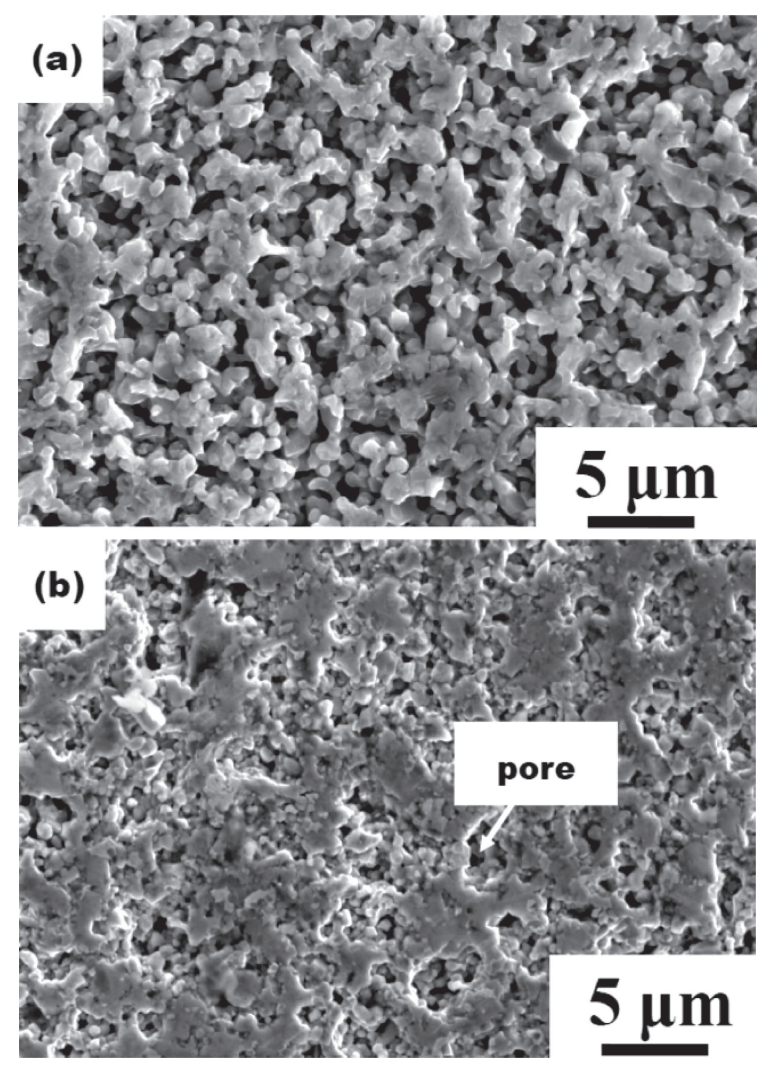

Fig. 7 Microstructure of sintered compacts; (a) As received fine powders compact, $C_{\mathrm{v}}=0.59$, (b) coarse MM 360 ks powders compact, $C_{\mathrm{v}}=2.06$.

both mean particle size and dispersion into account, the sinterability of the respective powder can be predicted more accurately and holistically.

\section{Conclusion}

This work has investigated the effect of dispersion in Particle Size Distribution (PSD) to sintering ability of $\mathrm{SiC}$ ceramics under constant sintering temperature. Mechanical milling was adopted to create various patterns of PSD. Mechanical milling has resulted in the reduction of particles aspect ratio for coarser particle and no significance change in finer particles. The usage of Coefficient of variation, $C_{\mathrm{v}}$, has demonstrated a reliable correlation for $\mathrm{SiC}$ sinter ability and thus was suggested as the most appropriate dispersion measure for PSD which do not hold identical mean values. In the range PSD investigated, the result showed that the sinterability of $\mathrm{SiC}$ ceramic improved with the increase of $C_{\mathrm{v}}$ value under constant sintering conditions.

\section{Acknowledgments}

This research was supported by the Japan Science and Technology Agency (JST) under a Collaborative Research Based on Industrial Demand "Heterogeneous Structure Control: Towards Innovative Development of Metallic Structural Materials", and by the Grant-in-Aid for Scientific Research on Innovative Area, Bulk Nanostructured Metals through MEXT, Japan (contract No. 22102004). These supports are gratefully acknowledged. 


\section{REFERENCES}

1) F. M. Varela-Feria, J. Martinez-Fernandez, A. R. de Arellano-Lopez and M. Singh: J. Eur. Ceram. Soc. 22 (2002) 2719-2725.

2) J. Sánchez-González, A. L. Ortiz, F. Guiberteau and C. Pascual: J. Eur. Ceram. Soc. 27 (2007) 3935-3939.

3) Q.-W. Huang and L. H. Zhu: Mater. Lett. 59 (2005) 1732-1735.

4) A. Maître, A. Vande Put, J. P. Laval, S. Valette and G. Trolliard: J. Eur. Ceram. Soc. 28 (2008) 1881-1890.

5) M. Singh and J. A. Salem: J. Eur. Ceram. Soc. 22 (2002) 2709-2717.

6) S. H. Lee: J. Ceram. Soc. Jpn. 119 (2011) 640-644.

7) A. Lara, A. L. Ortiz, A. Muñoz and A. Domínguez-Rodríguez: Ceram. Int. 38 (2012) 45-53.

8) A. Can, M. Hermann, D. S. McLachlan, I. Sigalas and J. Adler: J. Eur. Ceram. Soc. 26 (2006) 1707-1713.

9) M. F. Yan: Mater. Sci. Eng. 48 (1981) 53-72.

10) E. A. Barringer and H. K. Bowen: J. Am. Ceram. Soc. 65 (1982) C-199-C-201.
11) M. D. Sacks and T. Y. Tseng: J. Am. Ceram. Soc. 67 (1984) 532-537.

12) D. J. Sordelet and M. Akinc: J. Am. Ceram. Soc. 71 (1988) 1148-1153.

13) B. R. Patterson, V. D. Parkhe and J. A. Griffin: Mod. Des. Pow. Metall. 15 (1984) 279-288.

14) T. S. Yeh and M. D. Sacks: J. Am. Ceram. Soc. 71 (1988) C484-C487.

15) J. Ma and L. C. Lim: J. Eur. Ceram. Soc. 22 (2002) 2197-2208.

16) A. Wonisch, T. Kraft, M. Moseler and H. Riedel: J. Am. Ceram. Soc. 92 (2009) 1428-1434.

17) M. Hussain, Y. Oku, A. Nakahira and K. Niihara: Mater. Lett. 26 (1996) 177-184.

18) R. Johnson and P. Kuby: Elementary Statistics, (Thomson Brooks/ Cole., California, 2007) pp. 118-119

19) Y. Han, J. Fan, T. Liu, H. Cheng and J. Tian: Int. J. Ref. Met. H. Mater. 29 (2011) 743-750.

20) I. J. Shon and S. M. Kwak: J. Ceram. Proc. Res. 14 (2013) 694-699.

21) J. Sappakitkamjorn and S. Niwitpong: Int. J. Math. Comput. Nat. Phys. Eng. 7 (2013) 885-890.

22) J. D. Curto and J. C. Pinto: J. Appl. Stat. 36 (2009) 21-32. 